\title{
Neutrophil Elastase Contributes to Acute Lung Injury Induced by Bilateral Nephrectomy
}

\author{
Tomoko Ishii, ${ }^{*}$ Kent Doi, ${ }^{\star \dagger}$ Koji Okamoto, ${ }^{*}$ \\ Mitsuru Imamura, ${ }^{\ddagger}$ Makoto Dohi, ${ }^{\ddagger}$ \\ Kazuhiko Yamamoto, ${ }^{\ddagger}$ Toshiro Fujita, ${ }^{\star \dagger}$ \\ and Eisei Noiri* \\ From the Departments of Nephrology \& Endocrinology, * \\ Hemodialysis \& Apheresis, ${ }^{\dagger}$ and Allergy \& Rheumatology, ${ }^{\ddagger}$ \\ University Hospital, The University of Tokyo, Tokyo, Japan
}

Acute kidney injury (AKI) is a serious problem in critically ill patients of intensive care units. It has been reported previously that AKI can induce acute lung injury (ALI), as well as cause injuries to other remote organs, including the lungs. Patients with AKI complicated by ALI show remarkably high mortality. ALI is characterized by neutrophil infiltration into the lung. Neutrophil elastase (NE) is a key enzyme for tissue injury caused by activated neutrophils, such as occurs in ALI. Therefore, this study investigated the role of NE in AKI-induced ALI using a specific NE inhibitor, sivelestat sodium hydrate (ONO-5046), in a mouse bilateral nephrectomy model. Bilateral nephrectomy showed not only a remarkable increase in blood urea nitrogen levels, but also demonstrated neutrophil infiltration into the lung, increased pulmonary inflammatory cytokine expression [interleukin-6, neutrophil chemokine keratinocyte-derived chemokine, and tumor necrosis factor- $\alpha$ ], and protein leakage with early increases in both systemic and pulmonary NE activity. ONO-5046 treatment reduced NE activity and improved these pulmonary inflammatory responses. Additionally, ONO-5046-treated animals had longer survival times. These data demonstrate that increasing NE activity induces pulmonary inflammatory damage in a bilateral nephrectomy model. Blockade of NE activity will be a useful therapeutic strategy for ALI complications in AKI patients. (Am J Pathol 2010, 177:1665-1673; DOI: 10.2353/ajpath.2010.090793)

Acute lung injury (ALI) is a life-threatening condition that is frequently complicated with acute kidney injury (AKI), which is a serious condition in intensive care units. The ALI mortality is higher than $50 \%$ and increases with the development of other organ failure including AKI. ${ }^{1}$ One report described a mortality rate higher than $80 \%$ for patients with AKI complicated with ALI. ${ }^{2}$ Current prevention and treatment strategies for AKI cannot sufficiently improve the mortality of these severely ill patients. Although many basic and clinical researchers are investigating novel therapies for $\mathrm{AKI},{ }^{3}$ targeting of other organ damages caused by $A K I$ is also necessary for improving AKI outcomes.

Recently, it has been suggested that AKI influences other remote organs including the lungs. ${ }^{4-6}$ Experimental evidence indicates that AKI-induced ALI occurs not only by volume overload, but also through deleterious kidneylung interactions. Mechanisms of AKI-induced ALI include dysregulation of inflammatory reaction, innate immune response, oxidative stress, apoptosis, and soluble mediator metabolism. ${ }^{7}$ Rodent models of bilateral nephrectomy $(\mathrm{BNx})$ and renal ischemia reperfusion have been used to investigate the role of AKI in the pathogenesis of ALI. ${ }^{8-11}$ Ischemic AKI engenders increased pulmonary vascular permeability by down-regulating pulmonary epithelial sodium channel, Na,K-ATPase, and aquaporin-5. ${ }^{10}$ Ischemic AKI induced leukocyte accumulation and activation of inflammatory transcription factors such as nuclear factor- $\kappa \mathrm{B}$ and p38 mitogen-activated protein kinase in the lung, which was attenuated by an anti-inflammatory cytokine $\alpha$-melanocyte stimulating hormone. ${ }^{11}$ Faubel and colleagues ${ }^{5}$ demonstrated that $\mathrm{BNx}$ induced increases of multiple serum cytokines including interleukin (IL)-6 and lung injury characterized by neutrophil infiltration. Anti-inflammatory cytokine IL-10 attenuated pulmonary histological damage, bronchoalveolar lavage fluid (BALF) protein levels, and pulmonary myeloperoxidase activity. ${ }^{8}$

Supported by the BioBank Japan Project on the Implementation of Personalized Medicine \#3023168, Ministry of Education, Culture, Sports, Science and Technology (MEXT), Japan (E.N.), KAKEN-HI \#19590935, MEXT, Japan (E.N.), the Special Coordination Funds for Promoting Science and Technologies \#1200015, MEXT, Japan (E.N.), and KAKEN-HI \#21790795, MEXT, Japan (K.D.).

Accepted for publication June 8, 2010

Address reprint requests to Dr. Eisei Noiri, Department of Nephrology 107 Lab., University Hospital, The University of Tokyo, 7-3-1 Hongo, Bunkyo, Tokyo 113-8655, Japan. E-mail: noiri-tky@umin.ac.jp. 
Neutrophils play a central role in the pathogenesis of ALI by releasing several proteases and reactive oxygen species. ${ }^{12}$ Among them, neutrophil elastase (NE) is recognized as a potential target of therapeutic intervention. $\mathrm{NE}$ is a serine protease secreted by neutrophils during inflammation; it destroys not only bacteria but also host tissue. ${ }^{13,14}$ A specific NE inhibitor, sivelestat sodium hydrate (ONO-5046), is now approved for clinical use on ALI complicated with systemic inflammatory response syndrome in Japan. Although several clinical reports describe the protective effects of ONO-5046 on human ALI associated with systemic inflammatory response syndrome,$^{15-19}$ the literature includes no report of a clinical study evaluating the effect of ONO-5046 on ALI complicated with AKI. The present study is designed to elucidate the possible therapeutic effects of a specific NE inhibitor ONO-5046 on ALI induced by AKI using mouse BNx model. In addition to systemic and pulmonary NE activity and histological analysis of the lung, inflammatory cytokine expression in the lung was evaluated to clarify the role of $\mathrm{NE}$ in AKI-induced ALI.

\section{Materials and Methods}

\section{Animals and Surgical Protocol}

Eight- to ten-week-old male C57BL/6 mice, weighing 20 to $25 \mathrm{~g}$, were obtained from Japan SLC (Hamamatsu, Japan). Those mice were kept on a 12-hour light/dark cycle with free access to diet and water. All animal experiments were conducted in accordance with the $\mathrm{NIH}$ Guide for the Care and Use of Laboratory Animals (U.S. Department of Health and Human Services, Public Health Services, National Institutes of Health, $\mathrm{NIH}$ publication no. 86-23, 1985).

Animals were anesthetized by injection (i.p.) with a combination of ketamine hydrochloride and xylazine hydrochloride. The kidneys were exposed from the flank, dissected, and removed after the pedicles were ligated using 5-0 nylon sutures; alternatively, kidneys were isolated in sham surgeries. ONO-5046, provided by Ono Pharmaceutical Co., Ltd. (Osaka, Japan), was dissolved in normal saline and adjusted to $\mathrm{pH} 7.8$ with $\mathrm{Na}_{2} \mathrm{CO}_{3}$. In the $\mathrm{BNx}+\mathrm{ONO}-5046$ group, ONO-5046 was administered intraperitoneally at the dose of $50 \mathrm{mg} / \mathrm{kg} 11$ hours before the surgery and every 11 hours after (Figure 1). In the previous study on a mouse model of acute colitis,

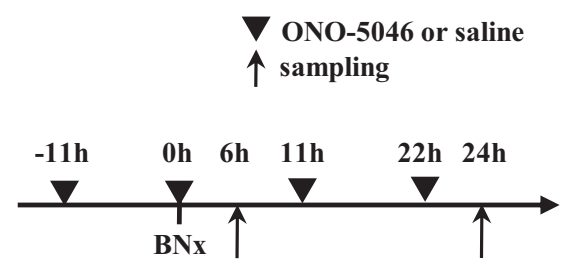

Figure 1. Protocol of ONO-5046 treatment. Acute lung injury was induced by bilateral nephrectomy. The experimental design of the BNx model and ONO-5046 treatment is shown. ONO-5046 treatment was performed 11 hours before surgery and every 11 hours after until death. Animals were killed 6 hours and 24 hours for blood and lung tissue sampling except survival analysis. treatment of ONO-5046 at the dose of $50 \mathrm{mg} / \mathrm{kg}$ twice a day improved histological damages and suppressed the NE activities. ${ }^{20}$ The same amount of saline was injected to the $\mathrm{BNx}+$ saline and the sham group. Animals were killed 6 hours or 24 hours after the surgery and blood and lung specimens were harvested for analyses, except for survival analysis. For survival analysis, animals were treated with ONO-5046 or saline every 11 hours until they died. Survival was assessed every 6 to 12 hours after the surgery.

\section{Measurement of Blood Urea Nitrogen, Aspartate Aminotransferase, and Alanine Aminotransferase}

The blood urea nitrogen was measured using the ureaseindophenol method with Urea NB (Wako Pure Chemical Industries Ltd., Osaka, Japan). The aspartate aminotransferase and alanine aminotransferase was measured using the POP-TOOS [pyruvate oxidase-N-ethyl-N-(2-hydroxy-3-sulfopropyl)-m-toluidine] method with Transaminase Cll-test Wako (Wako Pure Chemical Industries Ltd.). An absorbance 96-well plate reader (SpectraMAX Plus; Molecular Devices Corp., Sunnyvale, CA) was used with a wavelength of $570 \mathrm{~nm}$ (blood urea nitrogen) and $555 \mathrm{~nm}$ (aspartate aminotransferase and alanine aminotransferase).

\section{Histological Examination of Lung Tissue}

The lungs were resected from the mice after perfusion with PBS. The lungs were fixed with formalin and embedded in paraffin. Sections ( $3 \mu \mathrm{m}$ thick) were stained with Giemsa (Wako Pure Chemical Industries Ltd.). The number of neutrophils was determined in 10 randomly selected nonoverlapping fields at $\times 400$ magnification in each section of the individual mouse lung. Scores of respective lungs were averaged; scores of each animal were also averaged.

\section{Analysis of BALF}

The lungs were lavaged with $4 \times 0.5 \mathrm{ml}$ of PBS. The BALF was centrifuged $1500 \mathrm{rpm}$ at $4^{\circ} \mathrm{C}$ for 10 minutes, and the level of protein in the supernatant was measured using Bradford total protein assay (Bio-Rad Laboratories Inc., Hercules, CA). ${ }^{21}$ The cells were resuspended in $1 \mathrm{ml}$ of saline with $1 \%$ bovine serum albumin (Sigma-Aldrich, St. Louis, MO) and the total cell numbers were counted with a hemocytometer. Cytospin samples were prepared by centrifuging the suspensions at $350 \mathrm{rpm}$ for 10 minutes and cell differentials were determined by counting at least 300 leukocytes on Giemsa Stain (Wako Pure Chemical Industries, Ltd.). ${ }^{22,23}$ For neutrophil elastase activity measurement and Western blot analysis, BALF samples were condensed by spin condensing columns (Amicon Ultra; Millipore Corp., Billerica, MA). 


\section{Wet/Dry Lung Weight Ratios}

Lung wet/dry (W/D) weight ratios were measured as described previously. ${ }^{24}$ The lungs were surgically dissected, with removal of the trachea and main bronchi. Lungs were weighed and dried in an oven at $60^{\circ} \mathrm{C}$ for 48 hours. The W/D weight ratio was calculated to evaluate pulmonary edema.

\section{Measurement of NE Activity}

NE activity was determined as described previously. ${ }^{25}$ Briefly, plasma, supernatant of the lung homogenate, and BALF samples were incubated with $0.1 \mathrm{~mol} / \mathrm{L}$ Tris- $\mathrm{HCl}$ buffer ( $\mathrm{pH}$ 8.0) containing $0.5 \mathrm{~mol} / \mathrm{L} \mathrm{NaCl}$ and $1 \mathrm{mmol} / \mathrm{L}$ $\mathrm{N}$-methoxysuccinyl-Ala-Ala-Pro-Val $p$-nitroanilide, a highly specific synthetic substrate for $N E$, at $37^{\circ} \mathrm{C}$ for 24 hours and the liberated amount of $p$-nitroanilide was measured spectrophotometrically at $405 \mathrm{~nm}$. NE activity was calculated as the concentration of liberated $p$-nitroanilide. NE activity of lung homogenate and BALF was corrected with protein concentration.

\section{Western Blot Analysis}

Western blot analysis was performed as described previously. ${ }^{26}$ Briefly, $10 \mu \mathrm{g}$ proteins extracted from the lung or contained in BALF were separated on a 10 to $20 \%$ gradient sodium dodecyl sulfate-polyacrylamide gel and transferred to a polyvinylidene difluoride membrane (Amersham Biosciences Corp., Uppsala, Sweden). As a positive control, human leukocytes extracted from peripheral blood were used. ${ }^{27}$ Western blot analysis was performed using 1:150 diluted neutrophil elastase antibody ( $\mathrm{H}-57$; Santa Cruz Biotechnology Inc., Santa Cruz, CA) overnight at $4^{\circ} \mathrm{C}$. Subsequently, the chemiluminescent signal labeled using ECL Plus (Amersham Biosciences Corp.) was detected using a CCD camera system (LAS-4000 Mini; Fuji Photo Film Co. Ltd., Tokyo, Japan). The membrane was incubated at $50^{\circ} \mathrm{C}$ for 30 minutes in a stripping buffer to remove all probes. The reprobing procedure was performed further with the antibody to $\beta$-actin (Chemicon Co. Ltd., Temecula, CA). Densitometric analysis of bands compared with $\beta$-actin was performed using image software (Multi Gauge Ver. 3.0; Fuji Photo Film Co. Ltd.).

\section{Real-Time PCR Assay for IL-6, Keratinocyte-Derived Chemokine, and Tumor Necrosis Factor- $\alpha$ Expression in the Lung}

Total RNA was extracted from whole lung homogenates using Trizol (Invitrogen Corp., Carlsbad, CA). A High Capacity cDNA Reverse Transcription Kit (Applied Biosystems, Foster City, CA) was used according to the manufacturer's protocol to synthesize cDNA from total RNA with random primers. Lung transcription levels were assessed using real-time quantitative PCR with LightCycler Faststart DNA Master SYBR Green I (F. Hoffman La
Roche Ltd./Roche, Basel, Switzerland) and a PCR system (Prism 7000; Applied Biosystems), according to the manufacturer's instructions. The following primer-specific nucleotide sequences of IL-6 (sense 5'-TTCCATCCAGTTGCCTTCT-3' and antisense 5'-ATाCCACGATाCCCAGAG-3'), keratinocyte-derived chemokine (KC) (sense 5'-GGCTGGGATTCACCTCAAGAAC-3', antisense 5' -TGTGGCTATGACTTCGGTTTGG-3'), tumor necrosis factor (TNF)- $\alpha$ (sense 5'-ATCCGCGACGTGGAACTG-3' and antisense 5'-ACCGCCTGGAGTTCTGGAA-3'), and $\beta$-actin (sense 5'-CGCACCACTGGCATTGTCAT-3' and antisense 5'TTCTCCTTGATGTCACGCAC-3') were used. The PCR reaction conditions of $\mathrm{IL}-6, \mathrm{KC}$, and $\mathrm{TNF}-\alpha$ were $95^{\circ} \mathrm{C}$ for 10 minutes, followed by 45 cycles of $95^{\circ} \mathrm{C}$ for $10 \mathrm{~s}, 60^{\circ} \mathrm{C}$ for $10 \mathrm{~s}$, and $72^{\circ} \mathrm{C}$ for 30 seconds. Condition of $\beta$-actin was $95^{\circ} \mathrm{C}$ for 10 minutes, followed by 45 cycles of $95^{\circ} \mathrm{C}$ for $10 \mathrm{~s}, 53.5^{\circ} \mathrm{C}$ for $10 \mathrm{~s}$, and $72^{\circ} \mathrm{C}$ for 30 seconds. Quantification of gene expressions were calculated relative to $\beta$-actin. Amplification data were analyzed using software (Prism sequence detection ver. 2.1; Applied Biosystems).

\section{Statistical Analysis}

The results of statistical analyses are expressed as means \pm SEM. Differences between groups were analyzed for statistical significance using one-way analysis of variance followed by Tukey-Kramer test for multiple pairwise comparisons. $P<0.05$ was inferred as statistically significant. Survival was compared using a log-rank test. These calculations were performed using software (JMP 8.0; SAS Institute Inc., Cary, NC).

\section{Results}

\section{Effect of ONO-5046 on ALI Induced by BNx}

The protocol of $\mathrm{ALI}$ induced by $\mathrm{BNx}$ and ONO-5046 treatment is presented in Figure 1. A significant increase of blood urea nitrogen was observed in the $\mathrm{BNx}+$ saline and $\mathrm{BNx}+\mathrm{ONO}-5046$ group; no significant difference was found between them at any measurement time point of 2, 4, 8, and 24 hours. No significant difference was found in any body weight change or lung W/D weight ratio between the groups, which suggests that this $\mathrm{BNx}$ model had no remarkable volume overload. The ONO5046 treatment did not change the body weight and lung W/D weight ratio. Morphological analyses revealed no pulmonary interstitial edema in bilateral nephrectomized mice at 6 and 24 hours. Neutrophil infiltration evaluated by Giemsa stain was demonstrated in the BNx+saline group. Administration of ONO-5046 to sham-operated animals did not cause any neutrophil infiltration in the lung (Figure 2, A-C). The number of neutrophils was higher at 6 hours than at 24 hours after BNx and ONO5046 treatment decreased neutrophil infiltration at 6 hours and 24 hours (Figure 2, D).

BALF cellular content and protein levels were measured. BNx did not increase BALF cell counts including neutrophil compared with sham-operated animals (Fig- 

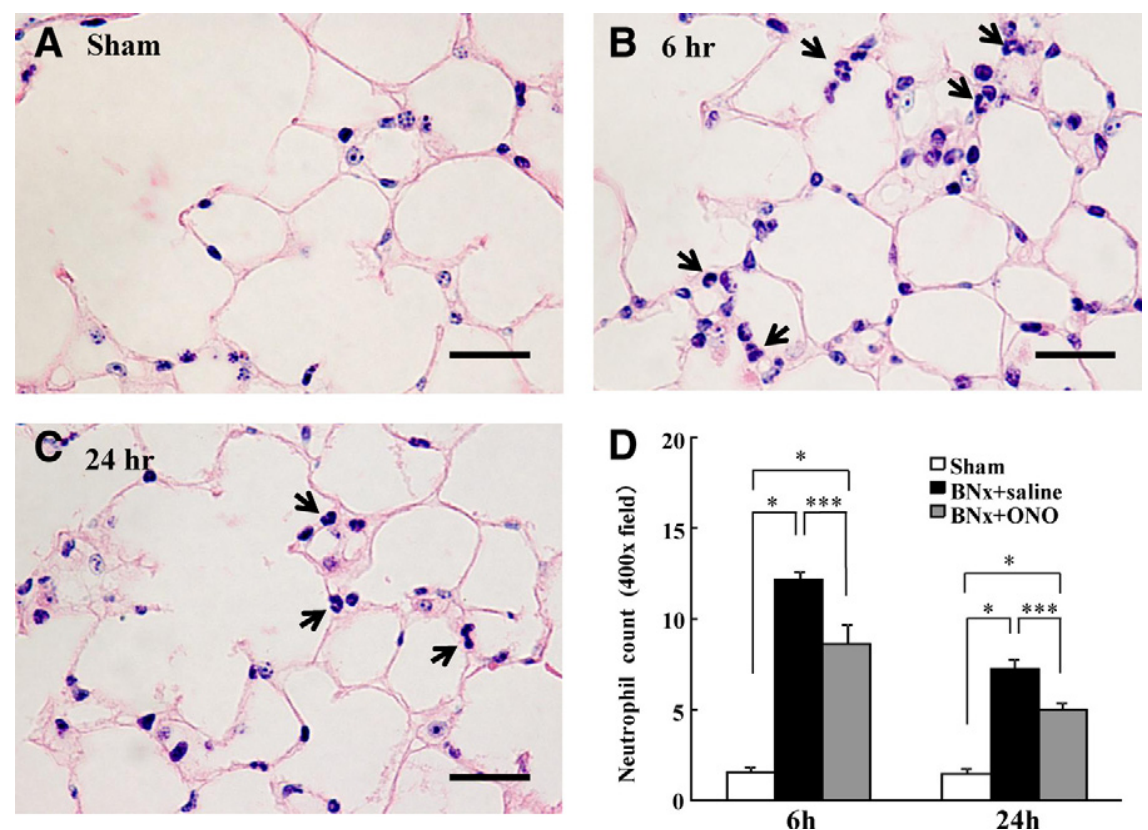

Figure 2. Neutrophil infiltration in ALI induced by BNx. Bilateral nephrectomy induced neutrophil infiltration in the lung. ONO-5046 treatment significantly reduced neutrophil numbers in the lung tissue. A-C: Neutrophil infiltration at 6 and 24 hours after the surgery was observed by $\mathrm{Gi}-$ emsa stain (arrows). Original magnification, $\times 400$. Scale bar $=50 \mu \mathrm{m}$. D: The neutrophil count in the lung was decreased significantly by ONO-5046 (ONO) treatment ( $n=5$ to 6 in each group; the number of neutrophils was determined in 10 randomly selected nonoverlapping fields in each section of the individual mouse lung.). ${ }^{*} P<0.001$ versus $\mathrm{BNx}+$ saline, ${ }^{* * * *} P<$ 0.05 versus sham ure $3 A$ ). No increase of BALF protein levels was found 6 hours after surgery. The BALF protein concentration in the $\mathrm{BNx}+$ saline group was significantly higher than that of the sham group at 24 hours. The ONO-5046 treatment engendered significantly lower BALF protein levels at 24 hours (Figure 3B).

\section{Survival and Liver Injury in ALI Induced by BNx}

The animals started to die 24 hours after the surgery of BNx. The ONO-5046 treatment significantly improved survival compared with saline injection (Figure 4). In accordance with the previous report, ${ }^{28} \mathrm{BNx}$ significantly increased plasma aspartate aminotransferase and alanine aminotransferase levels. The ONO-5046 treatment did not attenuate these liver enzymes (Figure 5, A and B).

\section{Neutrophil Elastase in ALI Induced by BNx}

Pulmonary and plasma NE activity increased at 6 hours after BNx and decreased to an equivalent level of sham after 24 hours. The BNx+ONO-5046 group showed significantly lower pulmonary (Figure 6A) and plasma (Figure 6B) NE activity at 6 hours. The NE activity in the BALF was not detected in all of the groups even when the BALF was condensed by spin condensing column. The amount of NE in the lung tissue was examined using Western blot analysis. Immunoreactive bands at approximately $30 \mathrm{kDa}$ were detected which corresponds to the molecular size of NE. NE protein in the lung was increased at 6 hours and 24 hours after BNx. The ONO-5046 treatment attenuated NE protein levels in the lung (Figure 7, A and B). No NE protein was detected in BALF by Western blot analysis (Figure 7A).

\section{Inflammatory Cytokine Expression in ALI Induced by BNx}

To evaluate pulmonary inflammatory responses in ALI induced by BNx, IL-6, KC, and TNF- $\alpha$ mRNA expressions in the lung were examined. Increased expressions of IL-6 (Figure 8A), KC (Figure 8B), and TNF- $\alpha$ (Figure 8C) were found in the $\mathrm{BNx}+$ saline group at 6 hours and 24 hours after BNx. These cytokine expressions were attenuated significantly by the ONO-5046 treatment (Figure 8, A-C).

\section{Discussion}

Acute lung injury complicated with $\mathrm{AKI}$ is highlighted by an unacceptably high mortality. Novel therapeutic strategies targeting this severe condition will improve critical care in intensive care units remarkably. This study demonstrated a specific NE inhibitor ONO-5046, which is now available for human ALI induced by systemic inflammatory response syndrome in Japan, improved ALI induced by $\mathrm{AKI}$ in a mouse model of BNx. Our data indicated that NE plays a crucial role in the pathogenesis of ALI induced by AKI.

NE is a serine protease located in neutrophil lysosome. Its role as a bactericidal agent has been established by generating mice lacking NE. ${ }^{29}$ The NE is secreted from activated neutrophils and contributes to tissue destruction in inflammatory diseases such as acute respiratory distress syndrome, ${ }^{30}$ lung emphysema, ${ }^{31}$ and rheumatoid arthritis. ${ }^{32}$ In normal conditions, NE is inactivated systemically and tightly by endogenous protease inhibitors such as $\alpha_{1}$-antitrypsin (AT) and $\alpha_{2}$-macroglobulin. However, inflammatory conditions inactivate these protease inhibitors by neutrophil-derived reactive oxygen 
A

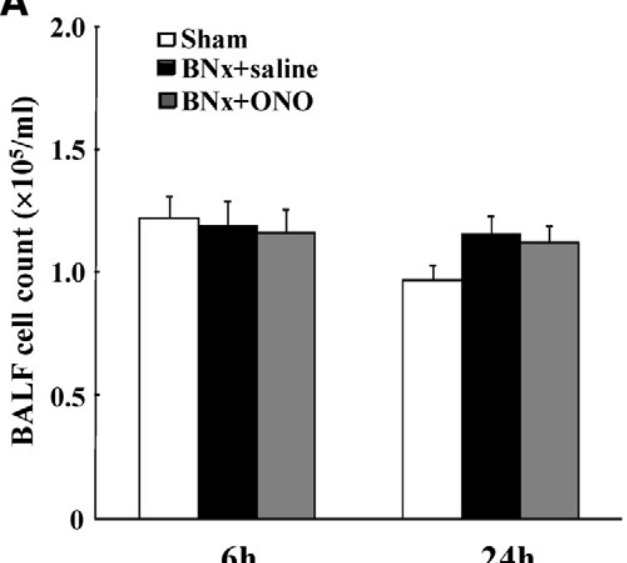

6h

24h

B

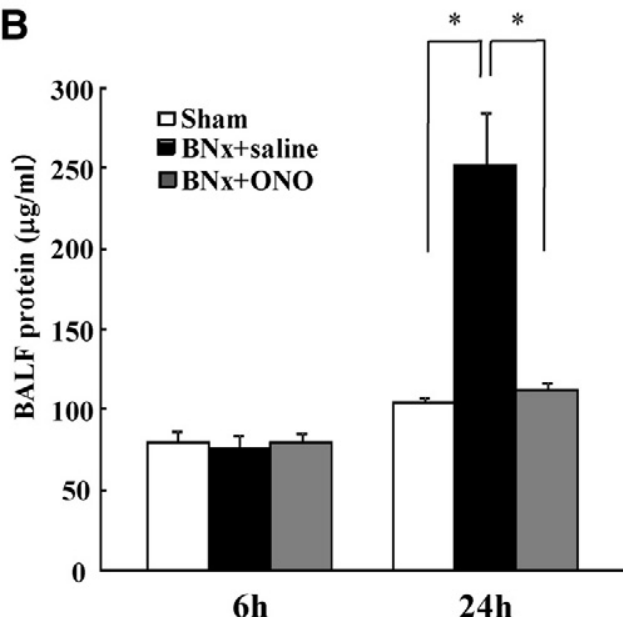

Figure 3. BALF cell count and protein concentration in ALI induced by BNx BALF obtained at 6 hours and 24 hours after bilateral nephrectomy was analyzed for evaluating pulmonary inflammation and vascular leakage. A: No significant difference was found in BALF cell count among the groups at 6 hours and 24 hours ( $n=5$ to 6 in each group). B: BALF protein concentration was increased at 24 hours and ONO-5046 (ONO) treatment significantly decreased BALF protein at 24 hours ( $n=5$ to 6 in each group). ${ }^{*} P<0.001$ versus $\mathrm{BNx}+$ saline.

species $^{12}$ and enable NE to have strong protease activity of destroying extracellular matrix such as elastin, collagen type I-IV, and proteoglycan. ${ }^{33}$ In the present study, systemic and pulmonary NE activity increased early after BNx (6 hours), which suggests that the acute decline of kidney function triggers inactivation of endogenous NE inhibitors. Reportedly, plasma elastase protein levels in dialyzed patients were increased even before dialysis treatment. ${ }^{34}$ Liver injury evaluated by blood aspartate aminotransferase and alanine aminotransferase levels was also found in the bilateral nephrectomized animals. $\alpha_{1}$-AT and $\alpha_{2}$-macroglobulin are produced by the liver and reduced synthesis of these endogenous NE inhibitors by the liver injury may partly contribute to NE activation in this model. Further investigations are necessary to clarify the regulatory mechanism of NE activation.

ONO-5046 attenuates lipopolysaccharide-induced pulmonary inflammation by inhibiting neutrophil infiltration and reducing lung vascular permeability in several animal models. ${ }^{35-37}$ In contrast to endogenous protease inhibitors, ONO-5046 assumed to be able to inhibit NE effec-

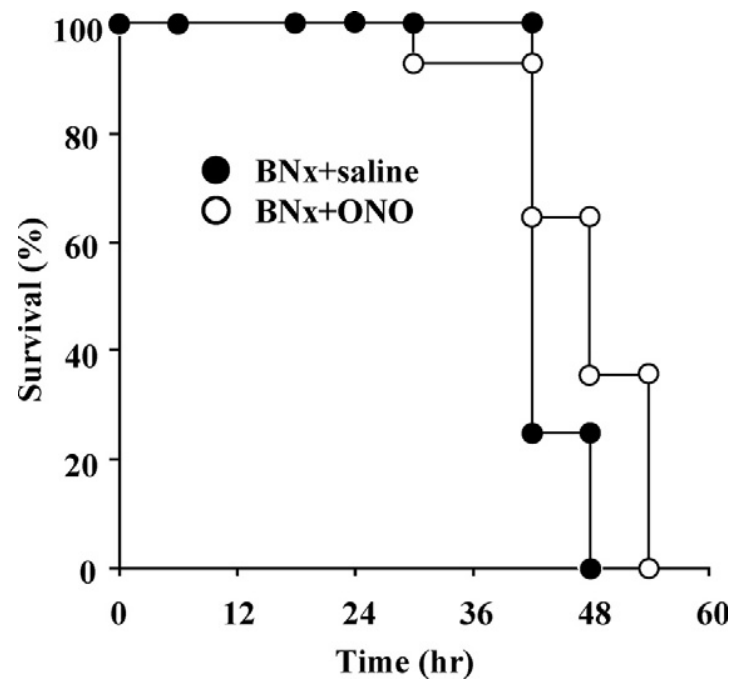

Figure 4. Effect of ONO-5046 on survival. Animals started to die 24 hours after bilateral nephrectomy and ONO-5046 (ONO) treatment showed a significant improvement of the survival after BNx compared with saline injection $(P<0.05$, by log-rank test). Closed circles indicate the $\mathrm{BNx}+$ saline group $(n=16)$ and open circles ONO-5046 treatment group $(n=14)$.

tively in inflammatory conditions because it is not inactivated by reactive oxygen species. ${ }^{38,39}$ The present study is the first report that shows the protective effect of $\mathrm{ONO}$ 5046 on AKI-induced ALI. Protective effects of ONO-
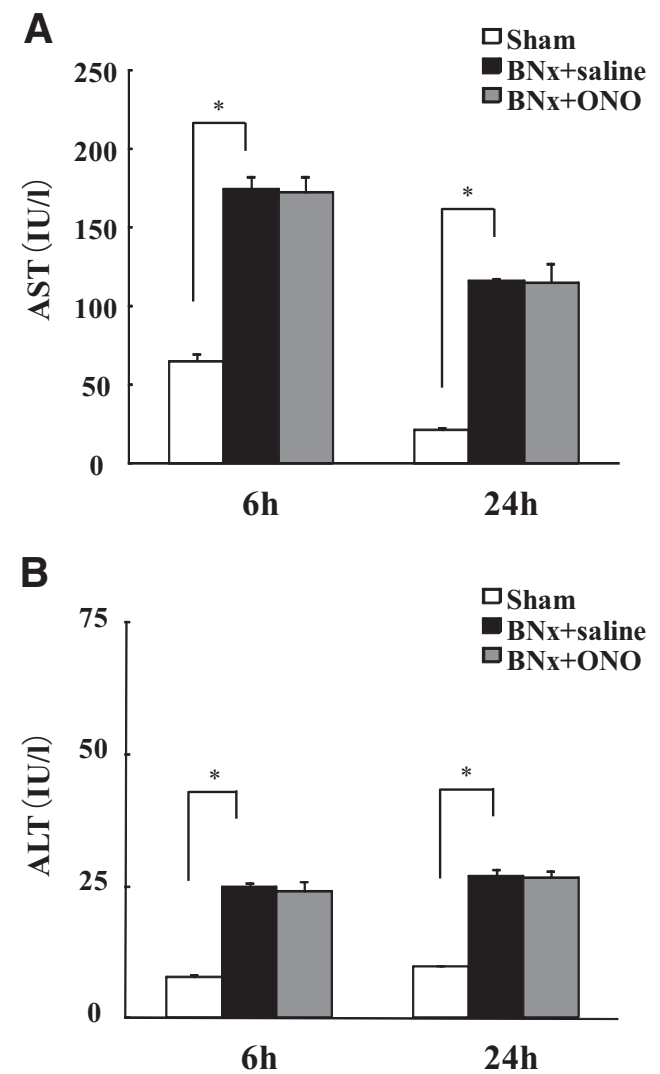

Figure 5. Liver injury in ALI induced by BNx. Liver injury was observed after bilateral nephrectomy. Plasma aspartate aminotransferase (A) and alanine aminotransferase (B) levels were significantly increased after the surgery. ONO-5046 (ONO) treatment did not improve liver injury $(n=7$ in each group). ${ }^{*} P<0.001$ versus sham. 
A

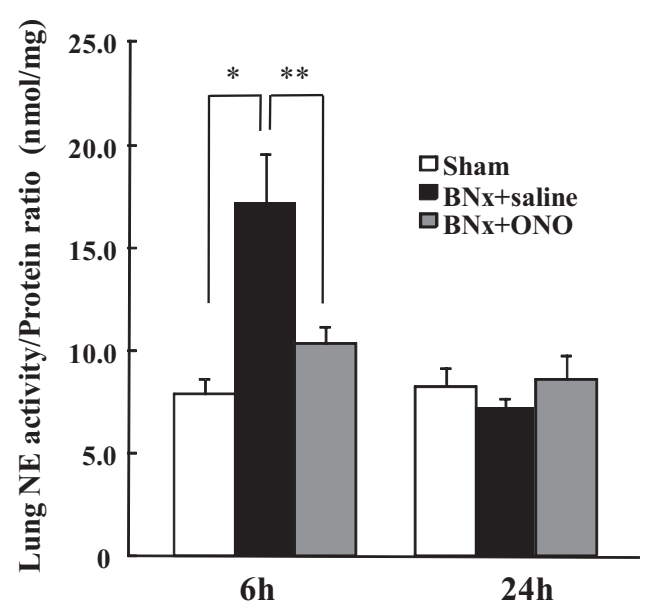

B

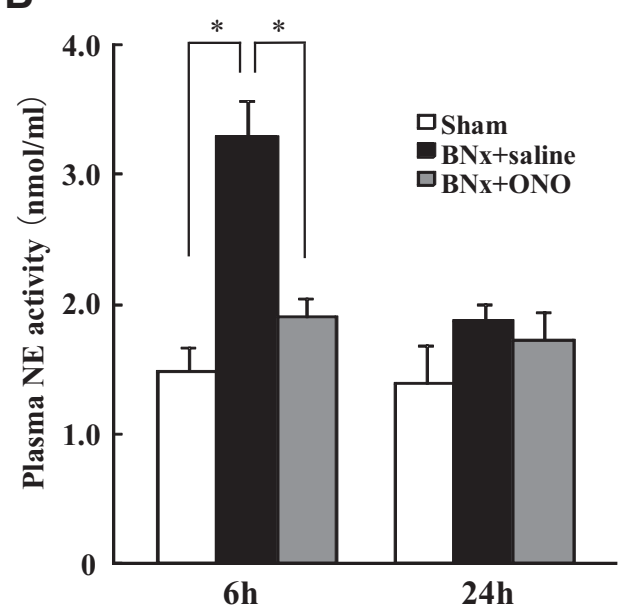

Figure 6. NE activity after AKI by BNx. To demonstrate the mechanism of protective effect of ONO-5046, lung (A) and plasma (B) NE activity after BNx were examined. $\mathrm{BNx}$ increased pulmonary and plasma NE activity at 6 hours and ONO-5046(ONO) treatment significantly decreased it $(n=5$ to 6 in each group). ${ }^{*} P<0.001,{ }^{* *} P<0.01$ versus $\mathrm{BNx}+$ saline.

5046 have also been demonstrated on neutrophil-mediated other organ injury models such as hippocampal neuronal damage after transient forebrain ischemia, ${ }^{40}$ neurological damage after spinal cord injury, ${ }^{41}$ collageninduced arthritis, ${ }^{42}$ and acute colitis. ${ }^{20}$ Although one randomized controlled trial including 492 patients demonstrated no improvement of ALI by ONO-5046, ${ }^{18}$ several small trials showed beneficial effects of ONO-5046 on ALI in critically ill patients. ${ }^{17,19}$ No clinical trial evaluating ONO-5046 on ALI complicated with AKI has been reported in the relevant literature. Our data may indicate the potential of NE inactivation for ALI treatment and induce new drug development for ALI complicated with AKI, which has an unacceptably high mortality rate. In addition, the therapeutic window of NE inactivation therapy should be considered for future clinical trials, because our data suggested the early intervention to NE activity by ONO-5046 is necessary to reduce AKI-induced ALI.

Results of this study showed a remarkable pulmonary neutrophil infiltration in ALI induced by BNx. Increased
A

6h

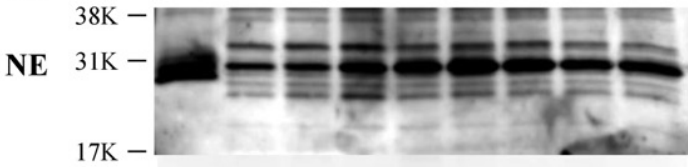

$\beta$-actin

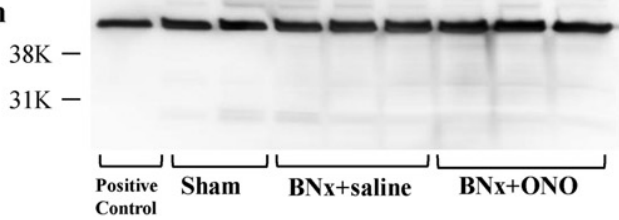

24h

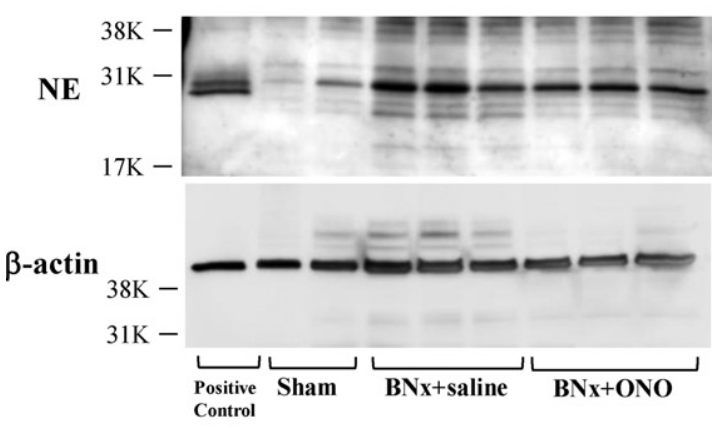

B

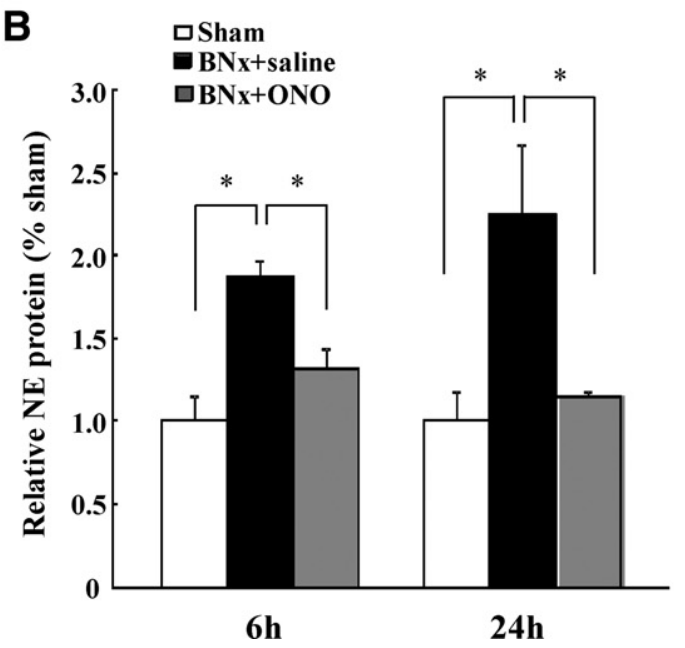

Figure 7. Pulmonary NE expression in ALI induced by BNx. A: The amount of NE protein was measured by Western blot analysis of lung homogenates. B: Densitometric analysis of immunoreactive bands compared with the density of $\beta$-actin demonstrated NE protein amount was significantly increased in the $\mathrm{BNx}+$ saline group, and attenuated by ONO-5046 (ONO) treatment $\left(n=6\right.$ in each group). ${ }^{*} P<0.05$ versus $\mathrm{BNx}+$ saline.

expression of $\mathrm{KC}$, which is a potent chemoattractant for neutrophils, was found in the lung tissue. NE induced IL-8 (human analogue of mouse $\mathrm{KC}$ ) expression in human bronchial epithelial cells. ${ }^{43}$ The KC production in zymosan-stimulated cremaster muscles of NE knockout mice was lower than in wild-type mice. ${ }^{44}$ We found attenuation of $\mathrm{KC}$ expression in the lung using ONO-5046 treatment. These data suggested that a vicious circle of NE-induced $\mathrm{KC}$ expression and subsequent neutrophil infiltration at least partly contribute to AKI-induced ALI; NE increased 
A

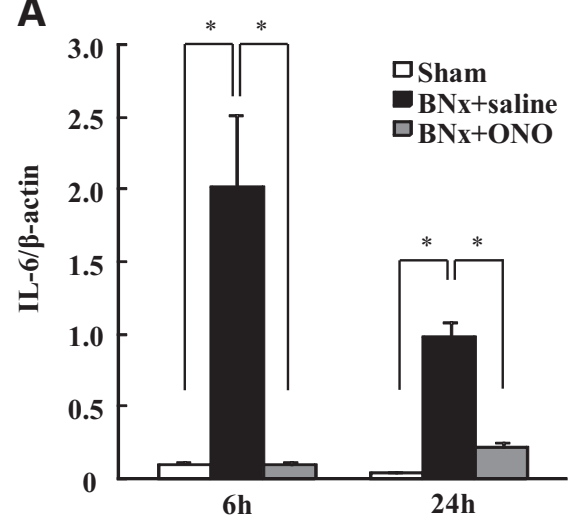

B

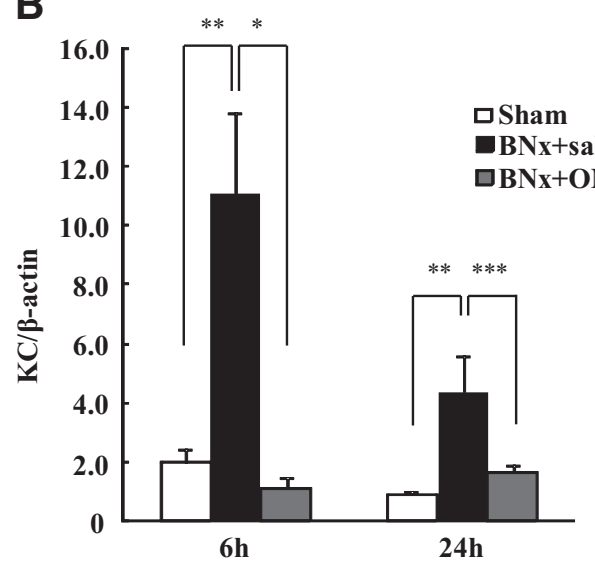

C

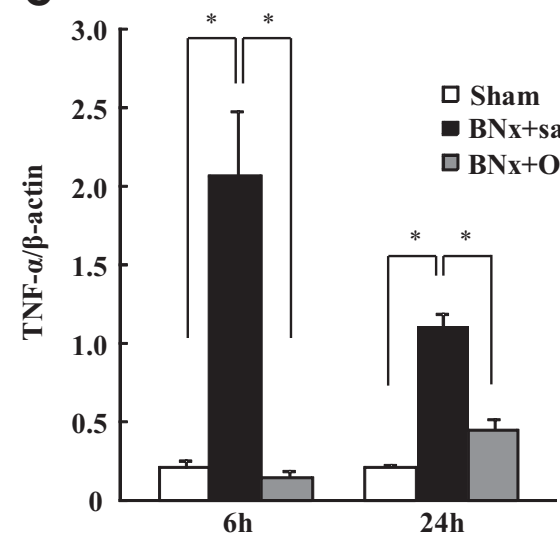

Figure 8. Inflammatory cytokine mRNA expression in ALI induced by BNx. Inflammatory responses induced by $\mathrm{BNx}$ were evaluated by several inflammatory cytokine expressions. mRNA expressions of IL-6 (A), KC (B), and TNF- $\alpha(\mathbf{C})$ in the lung were increased by $\mathrm{BNx}$ and ONO-5046 (ONO) treatment remarkably reduced these inflammatory cytokine expressions ( $n=5$ to 7 in each group). ${ }^{*} P<0.001,{ }^{* *} P<0.01,{ }^{*}{ }^{* * * *} P<0.05$ versus $\mathrm{BNx}+$ saline

$\mathrm{KC}$ expression in the lung, increased $\mathrm{KC}$, a potent chemoattractant, allowed neutrophil to infiltrate to the lung, and infiltrated neutrophil released NE and further increased $\mathrm{KC}$ levels.

We found a significant increase of NE protein in the lung, which was attenuated by ONO-5046 treatment. Reportedly, NE- $\alpha_{1}$-AT complex formation reduces elastase activity and NE- $\alpha_{1}$-AT complexes are cleared via serpinenzyme complex receptors. ${ }^{45,46}$ If the clearance of NE-
$\alpha_{1}$-AT complexes were blocked by ONO-5046, ONO5046 treatment would increase NE protein amount. However, $\alpha_{1}$-AT is larger and has higher potency compared with ONO-5046 ( $\alpha_{1}$-AT; $55 \mathrm{kDa}$, Ki $0.033 \mathrm{pM}$ versus ONO-5046; 0.5 kDa, Ki 29 nM). ${ }^{47,48} \mathrm{NE}$ inhibition by $\alpha_{1}$-AT is irreversible, whereas ONO-5046 inhibits NE activity reversibly. ${ }^{49}$ Therefore, it might be reasonable to discuss that $\alpha_{1}$-AT traps NE immediately and irreversibly in the injured lung, and ONO-5046 inhibited the excess $\mathrm{NE}$ that escaped from the natural defense system of $\alpha_{1}$-AT. In the situation that BNx induces NE production, ONO-5046 may not influence on the NE- $\alpha_{1}$-AT complex formation and clearance. It is true that there is no evidence whether ONO-5046 influences NE clearance and further investigations should be performed to clarify the influence of ONO-5046 on NE- $\alpha_{1}$-AT complex. It is of note that Endo et $\mathrm{al}^{50}$ reported ONO-5046 treatment on human septic ALI patients significantly reduced blood NE concentration.

Although one report showed that increased pulmonary vascular permeability in a rat ischemia reperfusion model was attenuated by an inhibitor of macrophage activation, ${ }^{51} \mathrm{BNx}$ did not induce any macrophage infiltration in the lung (data not shown). It can be speculated that activated macrophage might play a role in ALI in our model by releasing some humoral factors from other distant organs such as the liver.

In this study, we induced AKI not by ischemia reperfusion, but using BNx. As with renal ischemia, ischemia reperfusion models of other organs such as the liver, ${ }^{52}$ gut $^{53}$ and hind limb ${ }^{54}$ also cause ALI. Intestinal ischemia reperfusion injury induced tremendous lung injury, as characterized by lung edema, histopathological changes (alveolar congestion, hemorrhage, and infiltration of inflammatory cells), increased myeloperoxidase activity, and pro-inflammatory cytokines (IL-6 and TNF- $\alpha$ ) levels in the lung. ${ }^{55}$ In addition, renal ischemia reperfusion degrades other organ functions of the heart and brain. ${ }^{56,57}$ Therefore, it is difficult to distinguish the contribution of renal dysfunction itself on lung injury from the effects derived from non-renal organ injury or increased systemic oxidative stress. Because the BNx model induces abrupt and complete decline of renal function, it enables us to investigate the direct relation of renal dysfunction with $\mathrm{ALI}$ without considering the additive issues described above. ${ }^{5}$

In accordance with results described in a previous report, ${ }^{8}$ the mouse model of $A K I$-induced $A L I$ using BNx in the present study showed no volume overload or pulmonary edema. No significant difference was found between bilateral nephrectomized and sham-operated animals in terms of total body weight change or the lung W/D weight ratio. Severely ill patients of AKI-induced ALI frequently show lung edema possibly because they need fluid resuscitation to maintain their hemodynamics. However, we administered no fluid after the surgery to the animals. Although remarkable inflammatory responses including neutrophil infiltration, increased vascular permeability, and inflammatory cytokine expression were demonstrated, adding fluid as resuscitation will produce this AKI-induced ALI animal model closer to the clinical 
settings. Moreover, cell counts including neutrophil in BALF did not increase in this model. We could not detect any NE protein and activity in BALF. We observed neutrophil infiltration in the pulmonary interstitium but not in the alveolar cavities. This indicates the abrupt loss of renal function by BNx caused insufficient pulmonary inflammation to induce neutrophil migration into the alveolar cavity when compared with severe ALI models such as using oleic acid or LPS. ${ }^{58}$ Further investigation is necessary to evaluate whether ONO-5046 can protect AKI-induced ALI in more clinically relevant animal models.

In conclusion, we demonstrated that AKI induced by BNx caused lung injury highlighted by neutrophil infiltration; it also increased NE activity. Treatment with a specific NE inhibitor, ONO-5046, attenuated systemic and pulmonary NE activity and decreased neutrophil infiltration and inflammatory cytokine expression in the lung. These data indicate that NE plays a crucial role in AKIinduced ALI and that it can be a potential drug targeted to severe ALI complicated with AKI.

\section{Acknowledgments}

We are very grateful to Dr. Fumihiko Nakamura, Mami Haba, Kahoru Amitani (University of Tokyo), and Ryoji Matsumoto (Ono Pharmaceutical Co., Ltd.) for their skilled assistance.

\section{References}

1. Liu KD, Matthay MA: Advances in critical care for the nephrologist: acute lung injury/ARDS. Clin J Am Soc Nephrol 2008, 3:578-586

2. Chertow GM, Christiansen CL, Cleary PD, Munro C, Lazarus JM: Prognostic stratification in critically ill patients with acute renal failure requiring dialysis. Arch Intern Med 1995, 155:1505-1511

3. Waikar SS, Liu KD, Chertow GM: Diagnosis, epidemiology and outcomes of acute kidney injury. Clin J Am Soc Nephrol 2008, 3:844-861

4. Scheel PJ, Liu M, Rabb H: Uremic lung: new insights into a forgotten condition. Kidney Int 2008, 74:849-851

5. Faubel S: Pulmonary complications after acute kidney injury. Adv Chronic Kidney Dis 2008, 15:284-296

6. Feltes CM, Van Eyk J, Rabb H: Distant-organ changes after acute kidney injury. Nephron Physiol 2008, 109:80-84

7. Ko GJ, Rabb H, Hassoun HT: Kidney-lung crosstalk in the critically ill patient. Blood Purif 2009, 28:75-83

8. Hoke TS, Douglas IS, Klein CL, He Z, Fang W, Thurman JM, Tao Y, Dursun B, Voelkel NF, Edelstein CL, Faubel S: Acute renal failure after bilateral nephrectomy is associated with cytokine-mediated pulmonary injury. J Am Soc Nephrol 2007, 18:155-164

9. Klein CL, Hoke TS, Fang WF, Altmann CJ, Douglas IS, Faubel S: Interleukin-6 mediates lung injury following ischemic acute kidney injury or bilateral nephrectomy. Kidney Int 2008, 74:901-909

10. Rabb H, Wang Z, Nemoto T, Hotchkiss J, Yokota N, Soleimani M: Acute renal failure leads to dysregulation of lung salt and water channels. Kidney Int 2003, 63:600-606

11. Deng J, Hu X, Yuen PS, Star RA: Alpha-melanocyte-stimulating hormone inhibits lung injury after renal ischemia/reperfusion. Am J Respir Crit Care Med 2004, 169:749-756

12. Weiss SJ: Tissue destruction by neutrophils. N Engl J Med 1989, 320:365-376

13. Belaaouaj A, Kim KS, Shapiro SD: Degradation of outer membrane protein $A$ in Escherichia coli killing by neutrophil elastase. Science 2000, 289:1185-1188

14. Chua F, Laurent GJ: Neutrophil elastase: mediator of extracellular matrix destruction and accumulation. Proc Am Thorac Soc 2006, 3:424-427

15. Tamakuma S, Ogawa M, Aikawa N, Kubota T, Hirasawa H, Ishizaka A, Taenaka N, Hamada C, Matsuoka S, Abiru T: Relationship between neutrophil elastase and acute lung injury in humans. Pulm Pharmacol Ther 2004, 17:271-279

16. Okayama N, Kakihana $Y$, Setoguchi D, Imabayashi T, Omae T, Matsunaga A, Kanmura Y: Clinical effects of a neutrophil elastase inhibitor, sivelestat, in patients with acute respiratory distress syndrome. J Anesth 2006, 20:6-10

17. Inoue Y, Tanaka H, Ogura H, Ukai I, Fujita K, Hosotsubo H, Shimazu T, Sugimoto $H$ : A neutrophil elastase inhibitor, sivelestat, improves leukocyte deformability in patients with acute lung injury. J Trauma 2006, 60:936-943, discussion 943

18. Zeiher BG, Artigas A, Vincent JL, Dmitrienko A, Jackson K, Thompson BT, Bernard G: Neutrophil elastase inhibition in acute lung injury: results of the STRIVE study. Crit Care Med 2004, 32:1695-1702

19. Akamoto S, Okano K, Sano T, Yachida S, Izuishi K, Usuki H, Wakabayashi H, Suzuki Y: Neutrophil elastase inhibitor (sivelestat) preserves antitumor immunity and reduces the inflammatory mediators associated with major surgery. Surg Today 2007, 37:359-365

20. Morohoshi Y, Matsuoka K, Chinen H, Kamada N, Sato T, Hisamatsu T, Okamoto S, Inoue N, Takaishi H, Ogata H, Iwao Y, Hibi T: Inhibition of neutrophil elastase prevents the development of murine dextran sulfate sodium-induced colitis. J Gastroenterol 2006, 41:318-324

21. Nagatani K, Dohi M, To Y, Tanaka R, Okunishi K, Nakagome K, Sagawa K, Tanno Y, Komagata Y, Yamamoto K: Splenic dendritic cells induced by oral antigen administration are important for the transfer of oral tolerance in an experimental model of asthma. J Immunol 2006, 176:1481-1489

22. To Y, Dohi M, Tanaka R, Sato A, Nakagome K, Yamamoto K: Early interleukin 4-dependent response can induce airway hyperreactivity before development of airway inflammation in a mouse model of asthma. Lab Invest 2001, 81:1385-1396

23. Harada $\mathrm{H}$, Imamura M, Okunishi K, Nakagome $\mathrm{K}$, Matsumoto $\mathrm{T}$, Sasaki O, Tanaka R, Yamamoto K, Dohi M: Upregulation of lung dendritic cell functions in elastase-induced emphysema. Int Arch Allergy Immunol 2009, 149 Suppl 1:25-30

24. Folz RJ, Abushamaa AM, Suliman HB: Extracellular superoxide dismutase in the airways of transgenic mice reduces inflammation and attenuates lung toxicity following hyperoxia. J Clin Invest 1999, 103:1055-1066

25. Yoshimura K, Nakagawa S, Koyama S, Kobayashi T, Homma T: Roles of neutrophil elastase and superoxide anion in leukotriene B4-induced lung injury in rabbit. J Appl Physiol 1994, 76:91-96

26. Negishi K, Noiri E, Maeda R, Portilla D, Sugaya T, Fujita T: Renal L-type fatty acid-binding protein mediates the bezafibrate reduction of cisplatin-induced acute kidney injury. Kidney Int 2008, 73:1374-1384

27. Doi K, Noiri E, Nakao A, Fujita T, Kobayashi S, Tokunaga K: Functional polymorphisms in the vascular endothelial growth factor gene are associated with development of end-stage renal disease in males. J Am Soc Nephrol 2006, 17:823-830

28. Doi K, Yuen PS, Eisner C, Hu X, Leelahavanichkul A, Schnermann J Star RA: Reduced production of creatinine limits its use as marker of kidney injury in sepsis. J Am Soc Nephrol 2009, 20:1217-1221

29. Belaaouaj A, McCarthy R, Baumann M, Gao Z, Ley TJ, Abraham SN, Shapiro SD: Mice lacking neutrophil elastase reveal impaired host defense against gram negative bacterial sepsis. Nat Med 1998, $4: 615-618$

30. McGuire WW, Spragg RG, Cohen AB, Cochrane CG: Studies on the pathogenesis of the adult respiratory distress syndrome. J Clin Invest 1982, 69:543-553

31. Blue ML, Janoff A: Possible mechanisms of emphysema in cigarette smokers. Release of elastase from human polymorphonuclear leukocytes by cigarette smoke condensate in vitro. Am Rev Respir Dis 1978, 117:317-325

32. Schnebli HP, Christen P, Jochum M, Mallya RK, Pepys MB: Plasma levels of inhibitor-bound leukocytic elastase in rheumatoid arthritis patients. Adv Exp Med Biol 1984, 167:355-362

33. Havemann K, Gramse M: Physiology and pathophysiology of neutral proteinases of human granulocytes. Adv Exp Med Biol 1984, 167:1-20

34. Caimi G, Carollo C, Montana M, latrino R, Bondi B, Lo Presti R: Nitric oxide metabolites, leukocyte activation markers and oxidative status in dialyzed subjects. Blood Purif 2009, 27:194-198 
35. Kubo K, Kobayashi T, Hayano T, Koizumi T, Honda T, Sekiguchi M, Sakai A: Effects of ONO-5046, a specific neutrophil elastase inhibitor on endotoxin-induced lung injury in sheep. J Appl Physiol 1994, 77 : 1333-1340

36. Yasui S, Nagai A, Aoshiba K, Ozawa Y, Kakuta Y, Konno K: A specific neutrophil elastase inhibitor (ONO-5046.Na) attenuates LPS-induced acute lung inflammation in the hamster. Eur Respir J 1995, 8:1293-1299

37. Sakamaki F, Ishizaka A, Urano T, Sayama K, Nakamura H, Terashima T, Waki Y, Tasaka S, Hasegawa N, Sato K, Nakagawa N, Obata T, Kanazawa M: Effect of a specific neutrophil elastase inhibitor. ONO5046, on endotoxin-induced acute lung injury. Am J Respir Crit Care Med 1996, 153:391-397

38. Iwamura H, Moore AR, Willoughby DA: Interaction between neutrophil-derived elastase and reactive oxygen species in cartilage degradation. Biochim Biophys Acta 1993, 1156:295-301

39. Zeiher BG, Matsuoka S, Kawabata K, Repine JE: Neutrophil elastase and acute lung injury: prospects for sivelestat and other neutrophil elastase inhibitors as therapeutics. Crit Care Med 2002, 30:S281-287

40. Matayoshi H, Hirata T, Yamashita S, Ishida K, Mizukami Y, Gondo T, Matsumoto M, Sakabe T: Neutrophil elastase inhibitor attenuates hippocampal neuronal damage after transient forebrain ischemia in rats. Brain Res 2009, 1259:98-106

41. Tonai T, Shiba K, Taketani $\mathrm{Y}$, Ohmoto $\mathrm{Y}$, Murata $\mathrm{K}$, Muraguchi M, Ohsaki H, Takeda E, Nishisho T: A neutrophil elastase inhibitor (ONO5046) reduces neurologic damage after spinal cord injury in rats. J Neurochem 2001, 78:1064-1072

42. Kakimoto K, Matsukawa A, Yoshinaga M, Nakamura H: Suppressive effect of a neutrophil elastase inhibitor on the development of collagen-induced arthritis. Cell Immunol 1995, 165:26-32

43. McElvaney NG, Nakamura $H$, Birrer $P$, Hebert CA, Wong WL, Alphonso M, Baker JB, Catalano MA, Crystal RG: Modulation of airway inflammation in cystic fibrosis. In vivo suppression of interleukin-8 levels on the respiratory epithelial surface by aerosolization of recombinant secretory leukoprotease inhibitor. J Clin Invest 1992 90:1296-1301

44. Young RE, Thompson RD, Larbi KY, La M, Roberts CE, Shapiro SD, Perretti M, Nourshargh S: Neutrophil elastase (NE)-deficient mice demonstrate a nonredundant role for $\mathrm{NE}$ in neutrophil migration, generation of proinflammatory mediators, and phagocytosis in response to zymosan particles in vivo. J Immunol 2004, 172:4493-4502

45. Perlmutter DH, Glover GI, Rivetna M, Schasteen CS, Fallon RJ: Identification of a serpin-enzyme complex receptor on human hepatoma cells and human monocytes. Proc Natl Acad Sci USA 1990, 87: 3753-3757
46. Perlmutter DH, Joslin G, Nelson P, Schasteen C, Adams SP, Fallon $\mathrm{RJ}$ : Endocytosis and degradation of alpha 1-antitrypsin-protease complexes is mediated by the serpin-enzyme complex (SEC) receptor. J Biol Chem 1990, 265:16713-16716

47. Kawabata K, Hagio T, Matsuoka S: The role of neutrophil elastase in acute lung injury. Eur J Pharmacol 2002, 451:1-10

48. Gardiner PJ: Neutrophil elastase inhibitors. Eur Resp Rev 2002, 12:373-374

49. Nakayama Y, Odagaki Y, Fujita S, Matsuoka S, Hamanaka N, Naka $\mathrm{H}$, Toda M: Clarification of mechanism of human sputum elastase inhibition by a new inhibitor. ONO-5046, using electrospray ionization mass spectrometry. Bioorg Med Chem Lett 2002, 12:2349-2353

50. Endo S, Sato N, Yaegashi Y, Suzuki Y, Kojika M, Yamada Y, Yoshida $\mathrm{Y}$, Nakadate T, Aoki H, Inoue $\mathrm{Y}$ : Sivelestat sodium hydrate improves septic acute lung injury by reducing alveolar dysfunction. Res Commun Mol Pathol Pharmacol 2006, 119:53-65

51. Kramer AA, Postler G, Salhab KF, Mendez C, Carey LC, Rabb H: Renal ischemia/reperfusion leads to macrophage-mediated increase in pulmonary vascular permeability. Kidney Int 1999, 55:2362-2367

52. Colletti LM, Kunkel SL, Walz A, Burdick MD, Kunkel RG, Wilke CA, Strieter RM: Chemokine expression during hepatic ischemia/reperfusion-induced lung injury in the rat. The role of epithelial neutrophil activating protein. J Clin Invest 1995, 95:134-141

53. Caty MG, Guice KS, Oldham KT, Remick DG, Kunkel SI: Evidence for tumor necrosis factor-induced pulmonary microvascular injury after intestinal ischemia-reperfusion injury. Ann Surg 1990, 212:694-700

54. Seekamp A, Mulligan MS, Till GO, Smith CW, Miyasaka M, Tamatan T, Todd RF, 3rd, Ward PA: Role of beta 2 integrins and ICAM-1 in lung injury following ischemia-reperfusion of rat hind limbs. Am J Pathol 1993, 143:464-472

55. Dwivedi AJ, Wu R, Nguyen E, Higuchi S, Wang H, Krishnasastry K, Marini CP, Ravikumar TS, Wang P: Adrenomedullin and adrenomedullin binding protein-1 prevent acute lung injury after gut ischemia-reperfusion. J Am Coll Surg 2007, 205:284-293

56. Kelly KJ: Distant effects of experimental renal ischemia/reperfusion injury. J Am Soc Nephrol 2003, 14:1549-1558

57. Liu M, Liang Y, Chigurupati S, Lathia JD, Pletnikov M, Sun Z, Crow M, Ross CA, Mattson MP, Rabb H: Acute kidney injury leads to inflammation and functional changes in the brain. J Am Soc Nephrol 2008, 19:1360-1370

58. Matute-Bello G, Frevert CW, Martin TR: Animal models of acute lung injury. Am J Physiol Lung Cell Mol Physiol 2008, 295:L379-L399 